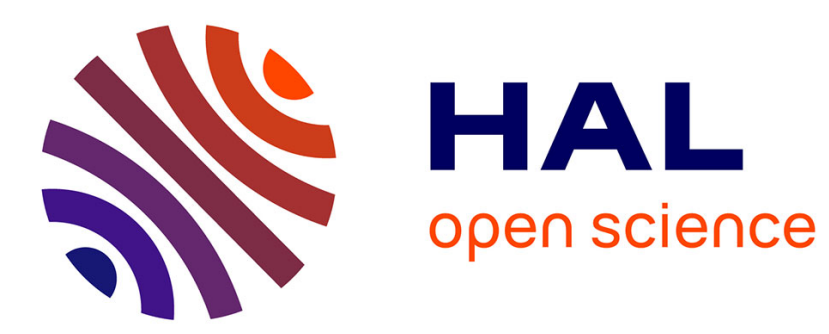

\title{
A characterization of the 2-additive Choquet integral through cardinal information
}

Brice Mayag, Michel Grabisch, Christophe Labreuche

\section{To cite this version:}

Brice Mayag, Michel Grabisch, Christophe Labreuche. A characterization of the 2-additive Choquet integral through cardinal information. Fuzzy Sets and Systems, 2011, 184 (1), pp.84-105. 10.1016/j.fss.2010.10.012 . halshs-00625708

\section{HAL Id: halshs-00625708 \\ https://shs.hal.science/halshs-00625708}

Submitted on 22 Sep 2011

HAL is a multi-disciplinary open access archive for the deposit and dissemination of scientific research documents, whether they are published or not. The documents may come from teaching and research institutions in France or abroad, or from public or private research centers.
L'archive ouverte pluridisciplinaire HAL, est destinée au dépôt et à la diffusion de documents scientifiques de niveau recherche, publiés ou non, émanant des établissements d'enseignement et de recherche français ou étrangers, des laboratoires publics ou privés. 


\section{Elsevier Editorial System(tm) for Fuzzy Sets and Systems Manuscript Draft}

Manuscript Number:

Title: A characterization of the 2-additive Choquet integral through cardinal information Article Type: Special Issue: Eurofuse 09

Keywords: MCDA, Choquet integral, 2-additive capacity, MACBETH, Preferences Corresponding Author: Mr. Brice MAYAG,

Corresponding Author's Institution: University of Paris 1

First Author: Brice MAYAG

Order of Authors: Brice MAYAG; Michel Grabisch; Christophe Labreuche 
To: Fuzzy Sets and Systems

Dear Sirs,

I would like to submit to Fuzzy Sets and Systems a paper of our research in the field of mathematics.

The paper is entitled: A characterization of the 2-additive Choquet integral through cardinal information

By Brice Mayag, Michel Grabisch and Christophe Labreuche.

Copy of the abstract:

In the context of Multiple criteria decision analysis, we present the necessary and sufficient conditions to represent a cardinal preferential information by the Choquet integral w.r.t. a 2additive capacity. These conditions are based on some complex cycles called cyclones.

Corresponding Author:

Brice Mayag

11 Route de Belleville

91190 Gif-sur-Yvette

France

Email: bmayag@yahoo.fr

I here certify that this paper consists of original, unpublished work which is not under consideration for publication elsewhere.

The original manuscript will be transferred following the instruction of the website of the Journal. I hope your favourable consideration for publication to Fuzzy Sets and Systems.

Sincerely,

Brice Mayag 
${ }^{*}$ Abstract

\begin{abstract}
In the context of Multiple criteria decision analysis, we present the necessary and sufficient conditions to represent a cardinal preferential information by the Choquet integral w.r.t. a 2-additive capacity. These conditions are based on some complex cycles called cyclones.
\end{abstract}

Keywords: MCDA, Choquet integral, 2-additive capacity, MACBETH methodology. 


\title{
A characterization of the 2-additive Choquet integral through cardinal information
}

\author{
Brice Mayag $^{\mathrm{a}, *}$, Michel Grabisch ${ }^{\mathrm{a}}$, Christophe Labreuche ${ }^{\mathrm{b}}$ \\ ${ }^{a}$ University of Paris I, Centre d'Economie de la Sorbonne, 106-112 Boulevard de \\ l'Hôpital, 75013 Paris, France \\ ${ }^{b}$ Thales $R$ \& $T$, Campus de Polytechnique, 1 avenue Augustin Fresnel, 91767 \\ Palaiseau cedex, France
}

\begin{abstract}
In the context of Multiple criteria decision analysis, we present the necessary and sufficient conditions to represent a cardinal preferential information by the Choquet integral w.r.t. a 2-additive capacity. These conditions are based on some complex cycles called cyclones.
\end{abstract}

Keywords: MCDA, Choquet integral, 2-additive capacity, MACBETH methodology.

\section{Introduction}

Multiple criteria decision analysis aims at representing the preferences of a decision maker over options or alternatives. The Choquet integral has been proved to be a versatile aggregation function to construct overall scores $[10,15$, 17] and arises as a generalization of the weighted sum, taking into account the interaction between criteria. In decision under uncertainty, as shown by the well-known Ellsberg's paradox [5, 13], the use of the expected utility model [41] is limited. Therefore some non-additive models like Choquet expected utility $[8,34]$ have been proposed in order to overcome the limitations of the expected utility model. In social welfare, it generalizes the Gini index [43].

The Choquet integral is defined w.r.t. a capacity (or nonadditive monotonic measure, or fuzzy measure), and can be thought as a generalization of the expected value, the capacity playing the role of a probability measure. Supposing a finite set of criteria denoted by $N$ and having at disposal some piece of information on the preference of the decision maker, a fundamental question in practice is to determine a capacity so that the Choquet integral can represent the revealed preference of the decision maker, if such a capacity exists at all. The fact that a capacity is defined on the power set of $N$ makes the

\footnotetext{
${ }^{*}$ Corresponding author.

Email addresses: bmayag@yahoo.fr (Brice Mayag), michel.grabisch@univ-paris1.fr (Michel Grabisch), christophe.labreuche@thalesgroup.com (Christophe Labreuche)
} 
problem exponentially complex, and thus practically intractable for large sets of criteria. For this reason, many simpler models have been proposed, where the capacity is restricted to some particular subspace. These are for example symmetric capacities (depending solely on the cardinality of subsets of $N$ ), as well as their generalization called $p$-symmetric capacities [25], $k$-additive capacities [15], whose Möbius transform lives on subsets of at most $k$ elements, decomposable capacities, [42] (including distorted probabilities), etc.

The concept of $k$-additive capacity seems to be of particular interest, since the value of $k$ is directly related to the complexity of the model (the number of subsets of at most $k$ elements) and it has a clear interpretation in many domains of decision making. In social welfare, the generalized Gini index proposed by [43] corresponds in fact to a Choquet integral w.r.t. a symmetric $k$-additive capacity, and has a very natural interpretation in terms of the weight the decision maker puts on envy in the society [14, 26]. For the 2 -additive case, it corresponds to a decision maker (DM) who is inequality averse in the sense that any Pigou-Dalton transfer increases his measure of welfare, wherever this transfer is applied on the income distribution. In multicriteria decision making, any interaction between two criteria can be represented and interpreted by a Choquet integral w.r.t. a 2 -additive capacity, but not more complex interaction. The Choquet integral w.r.t. a 2-additive capacity is very used in many applications such that the evaluation of discomfort in sitting position [16], the construction of performance measurement systems model in a supply chain context [4, 11], a conception of complex system for protection [31].

The above development suggests that the Choquet integral w.r.t. a 2additive capacity seems to be of particular interest, and offers a good compromise between flexibility of the model and complexity. Therefore, we focus in this paper on the 2-additive model.

In many situations, it is important for the Decision-Maker (DM) to construct a preference relation over the set of all alternatives $X$. Because it is not an easy task in most of practical situation (the cardinality of $X$ may be very large), we ask him to give, using pairwise comparisons, a cardinal information (a preferential information given with preference intensity) on a subset $X^{\prime} \subseteq X$ called reference set. In practice, these preferences are not sufficient to specify all the parameters of the model as the interaction between criteria because the reference set $X^{\prime}$ chosen by the DM is not specified. Therefore, we propose him to reason on some fictitious alternatives which represent concrete situations, are simple and easy to use, and are directly related to the parameters of the model. This approach is present in the MACBETH methodology for the computation of the weights for the arithmetic mean [2].

The use of Choquet integral requires to ensure the commensurateness between criteria i.e. one shall be able to compare any element of one point of view with any element of any other point of view. The only way to construct the utility functions with the Choquet integral uses the reference levels [21, 19]. As 
proved by [20] in his experiment of dictionaries read by some people, the attributes without reference level are less taken into account in the final decision.

The set $X^{\prime}$ we use in this paper is the set of binary alternatives or binary actions denoted by $\mathcal{B}$. A binary action is a fictitious alternative which takes either the neutral value (neither satisfactory nor unsatisfactory) $\mathbf{0}$ for all criteria, or the neutral value $\mathbf{0}$ for all criteria except for one or two criteria for which it takes the satisfactory value $\mathbf{1}$. The binary actions are used in many applications through the MACBETH methodology $[3,1,11]$. Since these alternatives have a very simple structure and make sense for the DM, he should have no difficulty to express preference on them. In [24], a characterization of the representation of an ordinal information (a preferential information containing only a strict preference and an indifference relations) on binary actions by a 2-additive Choquet integral have been proposed. This Characterization is based on two axioms, the classic cycles and the MOPI (MOnotonicity of Preferential Information) property which is related to the special kind of monotonicity induced by a 2-additive capacity. Our aim is to solve the following fundamental problem: Is the cardinal information of the decision maker representable by a Choquet integral w.r.t. a 2-additive capacity? If the answer is positive, one can extend the preference relation over the whole set $X$.

We present the necessary and sufficient conditions on the cardinal information for the existence of a 2-additive capacity such that the Choquet integral w.r.t. this capacity represents the preference of the DM. These conditions concern some cycles called cyclones in a directed graph where multiple edges are allowed between two vertices.

The basic material on the Choquet integral, binary actions and cardinal information are given in the next section. Section 3 studies the representation of a cardinal information by a Choquet integral w.r.t. a 2-additive capacity and give our main result. After Section 4 dedicated to proofs we need for our characterization, we propose in the last section some simple and necessary conditions which can be an alternative to cyclones to help the decision maker to deal with inconsistent cardinal information.

\section{Preliminaries}

\subsection{Notations and aim}

Let $N=\{1, \ldots, n\}$ be a finite set of $n$ criteria. An action (or alternative, option) $x=\left(x_{1}, \ldots, x_{n}\right)$ is identified with an element of the Cartesian product $X=X_{1} \times \cdots \times X_{n}$, where $X_{1}, \ldots, X_{n}$ represent the set of points of view or attributes. For a subset $A$ of $N$, the notation $z=\left(x_{A}, y_{N-A}\right)$ means that $z$ is defined by $z_{i}=x_{i}$ if $i \in A$, and $z_{i}=y_{i}$ otherwise.

We assume that, given two alternatives $x$ and $y$ the DM is able to judge the difference of attractiveness between $x$ and $y$ when he prefers strictly $x$ to $y$. Like in the MACBETH methodology [2], the difference of attractiveness will be provided under the form of semantic categories $d_{s}, s=1, \ldots, q$ defined 
so that, if $s<t$, any difference of attractiveness in the class $d_{s}$ is lower than any difference of attractiveness in the class $d_{t}$. MACBETH approach uses the following six semantic categories: $d_{1}=$ very weak, $d_{2}=$ weak, $d_{3}=$ moderate, $d_{4}=$ strong, $d_{5}=$ very strong, $d_{6}=$ extreme.

Our aim is to construct a preference relation over $X$. In practice (see [6, 23]) one can only ask to the DM pairwise comparisons of alternatives on a finite subset $X^{\prime}$ of $X, X^{\prime}$ having a small size. Hence we get a preference relation $\succsim_{X^{\prime}}$ on $X^{\prime}$. The question is then: how to construct a preference relation $\succsim X$ on $X$, so that $\succsim_{X}$ is an extension of $\succsim_{X^{\prime}}$ ? To this end, people usually suppose that $\succsim_{X}$ is representable by an overall utility function:

$$
x \succsim_{X} \quad y \Leftrightarrow F(U(x)) \geq F(U(y))
$$

where $U(x)=\left(u_{1}\left(x_{1}\right), \ldots, u_{n}\left(x_{n}\right)\right), u_{i}: X_{i} \rightarrow \mathbb{R}$ is called a utility function, and $F: \mathbb{R}^{n} \rightarrow \mathbb{R}$ is an aggregation function. Usually, we consider a family of aggregation functions characterized by a parameter vector $\theta$ (e.g., a weight distribution over the criteria). The parameter vector $\theta$ can be deduced from the knowledge of $\succsim_{X^{\prime}}$, that is, we determine the possible values of $\theta$ for which (1) is fulfilled over $X^{\prime}$. We study the case where $F$ is the Choquet integral w.r.t. a 2-additive capacity, then $X^{\prime}$ is the set of binary actions and the parameter vector is the 2 -additive capacity. The aim of this paper is to give necessary and sufficient conditions on $\succsim_{X^{\prime}}$ to be represented by a Choquet integral w.r.t. a 2-additive capacity. The model obtained in $X^{\prime}$ will be then automatically extended to $X$.

\subsection{Choquet integral w.r.t. a 2-additive capacity}

The Choquet integral $[17,15,18]$ is a well-known aggregation function used in multicriteria decision aiding when interactions between criteria occur. We are interested in the Choquet integral w.r.t a 2-additive capacity $[17,11]$. We define this notion below.

\section{Definition 2.1.}

1. A capacity on $N$ is a set function $\mu: 2^{N} \rightarrow[0,1]$ such that:

(a) $\mu(\emptyset)=0$

(b) $\mu(N)=1$

(c) $\forall A, B \in 2^{N},[A \subseteq B \Rightarrow \mu(A) \leq \mu(B)] \quad$ (monotonicity).

2. The Möbius transform (see [7]) of a capacity $\mu$ on $N$ is a function $m$ : $2^{N} \rightarrow \mathbb{R}$ defined by:

$$
m(T):=\sum_{K \subseteq T}(-1)^{|T \backslash K|} \mu(K), \forall T \in 2^{N} .
$$

When $m$ is given, it is possible to recover the original $\mu$ by the following expression:

$$
\mu(T):=\sum_{K \subseteq T} m(K), \forall T \in 2^{N} .
$$


Definition 2.2. A capacity $\mu$ on $N$ is 2-additive if

- For all subset $T$ of $N$ such that $|T|>2, m(T)=0$;

- There exists a subset $B$ of $N$ such that $|B|=2$ and $m(B) \neq 0$.

Notation. We simplify our notation for a capacity $\mu$ and its Möbius transform $m$ by using the following shorthand: $\mu_{i}:=\mu(\{i\}), \mu_{i j}:=\mu(\{i, j\}), m_{i}:=m(\{i\})$, $m_{i j}:=m(\{i, j\})$, for all $i, j \in N, i \neq j$. Whenever we use $i$ and $j$ together, it always means that they are different.

The following important lemma shows that a 2-additive capacity is entirely determined by the value of the capacity on the singletons $\{i\}$ and pairs $\{i, j\}$ of $2^{N}$ :

\section{Lemma 1.}

1. Let $\mu$ be a 2-additive capacity on $N$. We have

$$
\mu(K)=\sum_{\{i, j\} \subseteq K} \mu_{i j}-(|K|-2) \sum_{i \in K} \mu_{i}, \forall K \subseteq N,|K| \geq 2 .
$$

2. If the coefficients $\mu_{i}$ and $\mu_{i j}$ are given for all $i, j \in N$, then the necessary and sufficient conditions that $\mu$ is a 2-additive capacity are:

$$
\begin{gathered}
\sum_{\{i, j\} \subseteq N} \mu_{i j}-(n-2) \sum_{i \in N} \mu_{i}=1 \\
\mu_{i} \geq 0, \forall i \in N \\
\sum_{i \in A \backslash\{k\}}\left(\mu_{i k}-\mu_{i}\right) \geq(|A|-2) \mu_{k}, \forall A \subseteq N,|A| \geq 2, \forall k \in A .
\end{gathered}
$$

Proof. See [15].

Lemma 2. Let $\mu: 2^{N} \rightarrow \mathbb{R}_{+}$be a mapping such that

$$
\sum_{j \in A \backslash\{i\}}\left(\mu_{i j}-\mu_{j}\right) \geq(|A|-2) \mu_{i}, \forall A \subseteq N,|A| \geq 2, \forall i \in A .
$$

If $\exists i, j \in N$ such that $\mu_{i}>0$ or $\mu_{i j}>0$ then we have the following results:

1. $\sum_{\{i, j\} \subseteq N} \mu_{i j}-(n-2) \sum_{i \in N} \mu_{i}>0$. 
2. If we introduce

$$
\begin{aligned}
\alpha & :=\sum_{\{i, j\} \subseteq N} \mu_{i j}-(n-2) \sum_{i \in N} \mu_{i} \\
\mu_{\emptyset}^{\prime} & :=0 \\
\mu_{i}^{\prime} & :=\frac{\mu_{i}}{\alpha}, \forall i \in N \\
\mu_{i j}^{\prime} & :=\frac{\mu_{i j}}{\alpha}, \forall i, j \in N \\
\mu^{\prime}(K) & :=\sum_{\{i, j\} \subseteq K} \mu_{i j}^{\prime}-(|K|-2) \sum_{i \in K} \mu_{i}^{\prime}, \forall K \subseteq N,|K|>2,
\end{aligned}
$$

then $\mu^{\prime}$ is a 2-additive capacity on $N$.

Proof. See [24].

Given $x:=\left(x_{1}, \ldots, x_{n}\right) \in \mathbb{R}_{+}^{n}$, the expression of the Choquet integral w.r.t. a capacity $\mu$ is given by:

$$
C_{\mu}(x):=\sum_{i=1}^{n}\left(x_{\tau(i)}-x_{\tau(i-1)}\right) \mu(\{\tau(i), \ldots, \tau(n)\})
$$

where $\tau$ is a permutation on $N$ such that $x_{\tau(1)} \leq x_{\tau(2)} \leq \cdots \leq x_{\tau(n-1)} \leq x_{\tau(n)}$, and $x_{\tau(0)}:=0$.

A Choquet integral w.r.t. a 2-additive capacity $\mu$ is called for short a 2additive Choquet integral. Given $x:=\left(x_{1}, \ldots, x_{n}\right) \in \mathbb{R}_{+}^{n}$, the 2 -additive Choquet integral can be written also as follows (see [18]):

$$
C_{\mu}(x)=\sum_{i=1}^{n} v_{i} x_{i}-\frac{1}{2} \sum_{\{i, j\} \subseteq N} I_{i j}\left|x_{i}-x_{j}\right|
$$

where

1. The index $v_{i}$ given by

$$
v_{i}:=\sum_{K \subseteq N \backslash i} \frac{(n-|K|-1) !|K| !}{n !}(\mu(K \cup i)-\mu(K))
$$

represents the importance of the criterion $i$ and corresponds to the Shapley value of $\mu[37]$.

2. The index $I_{i j}$ given by

$$
I_{i j}:=\mu_{i j}-\mu_{i}-\mu_{j}
$$

represents the interaction between the two criteria $i$ and $j[15,30]$. 


\subsection{Binary actions and cardinal information}

We assume that the DM is able to identify for each criterion $i$ two reference levels:

1. A reference level $\mathbf{1}_{i}$ in $X_{i}$ which he considers as good and completely satisfying if he could obtain it on criterion $i$, even if more attractive elements could exist. This special element corresponds to the satisficing level in the theory of bounded rationality of Simon [38].

2. A reference level $\mathbf{0}_{i}$ in $X_{i}$ which he considers neutral on $i$. The neutral level is the absence of attractiveness and repulsiveness. The existence of this neutral level has roots in psychology [39], and is used in bipolar models like Cumulative Prospect Theory [40].

We set for convenience $u_{i}\left(\mathbf{1}_{i}\right)=1$ and $u_{i}\left(\mathbf{0}_{i}\right)=0$. For more details about these reference levels, see $[18,17]$.

We call a binary action or binary alternative, an element of the set

$$
\mathcal{B}=\left\{\mathbf{0}_{N},\left(\mathbf{1}_{i}, \mathbf{0}_{N-i}\right),\left(\mathbf{1}_{i j}, \mathbf{0}_{N-i j}\right), i, j \in N, i \neq j\right\} \subseteq X
$$

where

- $\mathbf{0}_{N}=\left(\mathbf{1}_{\emptyset}, \mathbf{0}_{N}\right)=: a_{0}$ is an action considered neutral on all criteria.

- $\left(\mathbf{1}_{i}, \mathbf{0}_{N-i}\right)=: a_{i}$ is an action considered satisfactory on criterion $i$ and neutral on the other criteria.

- $\left(\mathbf{1}_{i j}, \mathbf{0}_{N-i j}\right)=: a_{i j}$ is an action considered satisfactory on criteria $i$ and $j$ and neutral on the other criteria.

Using the Choquet integral, we get the following consequences:

1. For any capacity $\mu$,

$$
C_{\mu}\left(U\left(\left(\mathbf{1}_{A}, \mathbf{0}_{N-A}\right)\right)\right)=\mu(A), \forall A \subseteq N .
$$

2. For any 2-additive capacity, we have

$$
\begin{aligned}
C_{\mu}\left(U\left(a_{0}\right)\right) & =0 \\
C_{\mu}\left(U\left(a_{i}\right)\right) & =\mu_{i}=v_{i}-\frac{1}{2} \sum_{k \in N, k \neq i} I_{i k} \\
C_{\mu}\left(U\left(a_{i j}\right)\right) & =\mu_{i j}=v_{i}+v_{j}-\frac{1}{2} \sum_{k \in N, k \notin\{i, j\}}\left(I_{i k}+I_{j k}\right)
\end{aligned}
$$

The last two equations come from general relations between the capacity $\mu$ and interaction (see [15] for details). Generally the DM knows how to compare some alternatives using his knowledge of the problem, his experience, etc. 
These alternatives form a set of reference alternatives and allow to determine the parameters of a model (weights, utility functions, subjective probabilities,...) in the decision process (see [23] for more details). As shown by the previous equations (14), (15), (16) and Lemma 1, it should be sufficient to get some preferential information from the DM only on binary actions. To entirely determine the 2-additive capacity, this information is expressed by the following relations:

- $P=\{(x, y) \in \mathcal{B} \times \mathcal{B}$ : the DM strictly prefers $x$ to $y\}$,

- $I=\{(x, y) \in \mathcal{B} \times \mathcal{B}$ : the DM is indifferent between $x$ and $y\}$,

- For the semantic category " $d_{k}$ ", $k \in\{1, \ldots, q\}, P_{k}=\{(x, y) \in P$ such that DM judges the difference of attractiveness between $x$ and $y$ belonging to the class " $\left.d_{k} "\right\}$

Without loss of generality, we will suppose that all the relations $P_{k}$ are nonempty (we can always redefine the number $q$ when some $P_{k}$ are empty). The relation $P$ is irreflexive and asymmetric while $I$ is reflexive and symmetric.

Definition 2.3. The cardinal information on $\mathcal{B}$ is the structure $\left\{P, I, P_{1}, \ldots, P_{q}\right\}$.

The cardinal information is used also in the MACBETH methodology (see [2]). Now we will suppose $P$ to be nonempty for any cardinal information $\left\{P, I, P_{1}, \ldots, P_{q}\right\}$ ("non triviality axiom") and $P=P_{1} \cup P_{2} \cup \cdots \cup P_{q}$.

\section{The representation of the cardinal information by the Choquet integral}

\subsection{The representation}

A cardinal information $\left\{P, I, P_{1}, \ldots, P_{q}\right\}$ is said to be representable by a 2-additive Choquet integral if there exists a 2-additive capacity $\mu$ such that:

1. $\forall x, y \in \mathcal{B}, x P y \Rightarrow C_{\mu}(U(x))>C_{\mu}(U(y))$,

2. $\forall x, y \in \mathcal{B}, x I y \Rightarrow C_{\mu}(U(x))=C_{\mu}(U(y))$,

3. $\forall x, y, z, w \in \mathcal{B}, \forall s, t \in\{1, \ldots, q\}$ s.t. $s<t,\left[\begin{array}{c}(x, y) \in P_{t} \\ (z, w) \in P_{s}\end{array}\right\} \Rightarrow C_{\mu}(U(x))-$ $\left.C_{\mu}(U(y))>C_{\mu}(U(z))-C_{\mu}(U(w))\right]$

Given a cardinal information $\left\{P, I, P_{1}, \ldots, P_{q}\right\}$, we look for the necessary and sufficient conditions on $\mathcal{B}$ for which $\left\{P, I, P_{1}, \ldots, P_{q}\right\}$ is representable by a 2 additive Choquet integral. By using the monotonicity constraints of a 2 -additive capacity and Lemmas 1 and 2, this problem is equivalent to look for a function 
$f: \mathcal{B} \rightarrow \mathbb{R}_{+}$satisfying the following equations:

$$
\begin{aligned}
& \forall x, y \in \mathcal{B}, x P y \Rightarrow f(x)>f(y) \\
& \forall x, y \in \mathcal{B}, x I y \Rightarrow f(x)=f(y) \\
& \forall x, y, z, w \in \mathcal{B},\left[(x, y) P^{Q}(z, w) \Rightarrow f(x)-f(y)>f(z)-f(w)\right] \\
& f\left(a_{0}\right)=0 \\
& \forall i \in N, f\left(a_{i}\right) \geq 0 \\
& \forall A \subseteq N,|A| \geq 2, \forall i \in A, \sum_{j \in A \backslash\{i\}}\left(f\left(a_{i j}\right)-f\left(a_{j}\right)\right) \geq(|A|-2) f\left(a_{i}\right)
\end{aligned}
$$

The following lemmas show that the six previous conditions can be rewritten by introducing the thresholds $\sigma_{k}, k \in\{1, \ldots, q\}$ and $\sigma_{i j}^{i}, i, j \in N, i \neq j$.

Lemma 3. Let $\left\{P, I, P_{1}, \ldots, P_{q}\right\}$ be a cardinal information and $f: \mathcal{B} \rightarrow \mathbb{R}_{+}$. $f$ satisfies the conditions (17), (18), (19)

$\exists(q-1)$ real numbers $\sigma_{1}, \sigma_{2}, \ldots, \sigma_{q-1}$ such that

$$
\begin{aligned}
& \forall(x, y) \in I: f(x)=f(y), \\
& \forall k \in\{1, \ldots, q\}, \forall(x, y) \in P_{k}: \sigma_{k-1}<f(x)-f(y), \quad\left(\sigma_{0}:=0\right) \\
& \forall k \in\{1, \ldots, q-1\}, \forall(x, y) \in P_{k}: f(x)-f(y)<\sigma_{k} .
\end{aligned}
$$

Proof. see [2].

Lemma 4. Let $\left\{P, I, P_{1}, \ldots, P_{q}\right\}$ be a cardinal information and $f: \mathcal{B} \rightarrow \mathbb{R}_{+}$. $f$ satisfies the conditions (20), (21) and (22)

there exists nonnegative numbers $\sigma_{i j}^{i}, i, j \in N, i \neq j$ such that

$$
\begin{aligned}
& f\left(a_{0}\right)=0, \\
& \forall i \in N, f\left(a_{i}\right) \geq 0, \\
& \forall i, j \in N, i \neq j, f\left(a_{i j}\right)-f\left(a_{j}\right) \geq \sigma_{i j}^{i}, \\
& \forall A \subseteq N,|A| \geq 3, \forall i \in A, \sum_{j \in A \backslash\{i\}} \sigma_{i j}^{i} \geq(|A|-2) f\left(a_{i}\right) .
\end{aligned}
$$

Proof.

- $(\Leftarrow)$ We suppose that there exists $\sigma_{i j}^{i}, i, j \in N, i \neq j$ such that the conditions (28) and (29) are satisfied. We have for $i, j \in N, f\left(a_{i j}\right)-f\left(a_{j}\right) \geq$ $\sigma_{i j}^{i} \geq 0$. Using (28) and (29), we have for $A \subseteq N,|A| \geq 3$ and $i \in A$, $\sum_{j \in A \backslash\{i\}}\left(f\left(a_{i j}\right)-f\left(a_{j}\right)\right) \geq \sum_{j \in A \backslash\{i\}} \sigma_{i j}^{i} \geq(|A|-2) f\left(a_{i}\right)$. 
- $(\Rightarrow)$ Conversely it is sufficient to take $\sigma_{i j}^{i}=f\left(a_{i j}\right)-f\left(a_{j}\right), i, j \in N, i \neq j$ in order to satisfy the conditions (26), (27), (28) and (29) when (21) and (22) are fulfilled.

Corollary 1. Let $\left\{P, I, P_{1}, \ldots, P_{q}\right\}$ be a cardinal information and $f: \mathcal{B} \rightarrow \mathbb{R}_{+}$.

$f$ satisfies the conditions (17) to (22)

there exists $(q-1)$ real numbers $\sigma_{1}, \sigma_{2}, \ldots, \sigma_{q-1}$ and nonnegative numbers $\sigma_{i j}^{i}, \quad i, j \in N, i \neq j$ such that

$$
\begin{aligned}
& \forall(x, y) \in I: f(x)=f(y), \\
& \forall k \in\{1, \ldots, q\}, \forall(x, y) \in P_{k}: \sigma_{k-1}<f(x)-f(y), \quad\left(\sigma_{0}:=0\right) \\
& \forall k \in\{1, \ldots, q-1\}, \forall(x, y) \in P_{k}: f(x)-f(y)<\sigma_{k}, \\
& f\left(a_{0}\right)=0, \\
& \forall i, j \in N, i \neq j: f\left(a_{i j}\right)-f\left(a_{j}\right) \geq \sigma_{i j}^{i}, \\
& \forall A \subseteq N,|A| \geq 3, \forall i \in A: 0 \leq f\left(a_{i}\right) \leq \frac{1}{|A|-2} \sum_{j \in A \backslash\{i\}} \sigma_{i j}^{i} .
\end{aligned}
$$

Proof. It is a consequence of Lemmas 3 et 4.

Remark 1. If the equations (30), (31), (32) are satisfied then the real numbers $\sigma_{1}, \sigma_{2}, \ldots, \sigma_{q-1}$ are nonnegative.

\subsection{The binary relations $M$ and $M_{A}$ on $\mathcal{B}$}

To solve our problem, we introduce the following binary relations on $\mathcal{B}$ :

1. The relation $M=\left\{\left(a_{i j}, a_{i}\right), i, j \in N, i \neq j\right\}$ which models the natural monotonicity constraints $\mu_{i j} \geq \mu_{i}, i, j \in N$ for a capacity $\mu$ (see equation $(7))$, and translates the condition $f\left(a_{i j}\right)-f\left(a_{j}\right) \geq \sigma_{i j}^{i}$ in the previous Corollary $((34))$.

2. The relations $M_{A}=\left\{\left(a_{0}, a_{i}\right), i \in A\right\}, \forall A \subseteq N$ such that $|A| \geq 3$. These relations are defined in order to take into account, as it will be shown in the proof of Lemma 5 , the monotonicity and nonnegative conditions grouped in (35). Therefore they have been defined for any subset of criteria with a cardinality greater than three. Specifically, the condition

$f\left(a_{i}\right) \leq \frac{1}{|A|-2} \sum_{j \in A \backslash\{i\}} \sigma_{i j}^{i}$, which is equivalent to $f\left(a_{0}\right)-f\left(a_{i}\right) \geq-\frac{1}{|A|-2} \sum_{j \in A \backslash\{i\}} \sigma_{i j}^{i}$ when $f$ exists $\left(f\left(a_{0}\right)=0\right.$ in this case), will be model by the relation $M_{A}$. On the other hand, the condition $0 \leq f\left(a_{i}\right)$ which is equivalent to $f\left(a_{i}\right)-f\left(a_{0}\right) \geq 0$ will be modeled by $M_{A}^{-1}$, the converse of $M_{A}$. 


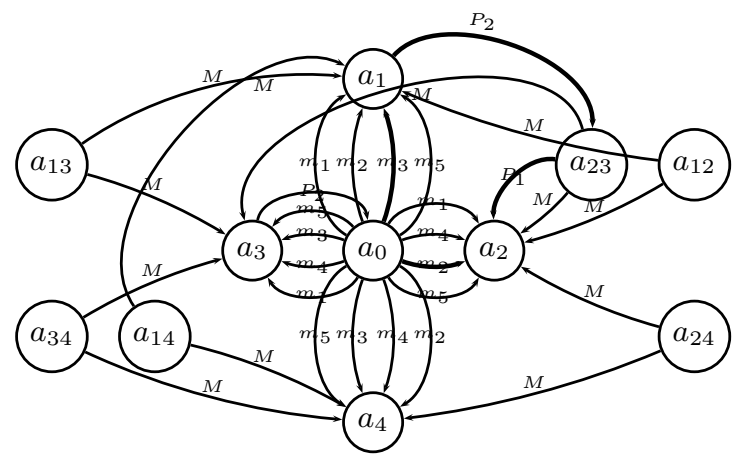

Figure 1: Graph of binary relations $P_{1}, P_{2}, M$ and $M_{A}$ on $\mathcal{B}$ with $N=\{1,2,3,4\}, M_{\{1,2,3\}}=$ $m_{1}, M_{\{1,2,4\}}=m_{2}, M_{\{1,3,4\}}=m_{3}, M_{\{2,3,4\}}=m_{4}$ and $M_{\{1,2,3,4\}}=m_{5}$

Let us give a simple example where these two binary relations are presented:

Example 1. Let be $N=\{1,2,3,4\}$. We have $M=\left\{\left(a_{12}, a_{1}\right),\left(a_{12}, a_{2}\right),\left(a_{13}, a_{1}\right)\right.$, $\left.\left(a_{13}, a_{3}\right),\left(a_{14}, a_{1}\right),\left(a_{14}, a_{4}\right),\left(a_{23}, a_{2}\right),\left(a_{23}, a_{3}\right),\left(a_{24}, a_{2}\right),\left(a_{24}, a_{4}\right),\left(a_{34}, a_{3}\right),\left(a_{34}, a_{4}\right)\right\}$, $M_{\{1,2,3\}}=: m_{1}=\left\{\left(a_{0}, a_{1}\right),\left(a_{0}, a_{2}\right),\left(a_{0}, a_{3}\right)\right\}, M_{\{1,2,4\}}=: m_{2}=\left\{\left(a_{0}, a_{1}\right)\right.$, $\left.\left(a_{0}, a_{2}\right),\left(a_{0}, a_{4}\right)\right\}, M_{\{1,3,4\}}=: m_{3}=\left\{\left(a_{0}, a_{1}\right),\left(a_{0}, a_{3}\right),\left(a_{0}, a_{4}\right)\right\}, M_{\{2,3,4\}}=:$ $m_{4}=\left\{\left(a_{0}, a_{2}\right),\left(a_{0}, a_{3}\right),\left(a_{0}, a_{4}\right)\right\}$ and $M_{\{1,2,3,4\}}=: m_{5}=\left\{\left(a_{0}, a_{1}\right),\left(a_{0}, a_{2}\right),\left(a_{0}, a_{3}\right)\right.$, $\left.\left(a_{0}, a_{4}\right)\right\}$. We assume also that $P_{1}=\left\{\left(a_{23}, a_{2}\right)\right\}$ and $P_{2}=\left\{\left(a_{1}, a_{23}\right) ;\left(a_{4}, a_{0}\right)\right\}$. All these relations are represented in the graph ${ }^{1}$ of the Figure 1

Notation: Given a cardinal information $\left\{P, I, P_{1}, \ldots, P_{q}\right\}$, we will use the notation below:

$$
\begin{aligned}
& \mathcal{P}^{\prime}=P \cup\left(\bigcup_{k=1}^{q-1} P_{k}^{-1}\right) \\
& \mathcal{R}=I \cup \mathcal{P}^{\prime} \cup M \cup\left(\bigcup_{\substack{A \subseteq N \\
|A| \geq 3}}\left(M_{A} \cup M_{A}^{-1}\right)\right)
\end{aligned}
$$

where for a general binary relation $R, R^{-1}=\{(y, x) \in \mathcal{B} \times \mathcal{B}: \quad(x, y) \in R\}$. The relations $\mathcal{P}, \mathcal{P}^{\prime}$ and $\mathcal{R}$ are binary relations on $\mathcal{B}$. Using all these binary relations, we define in the next section the main property for the characterization of a representation of a cardinal information by a Choquet integral w.r.t. a 2additive capacity.

\subsection{2-additive balanced cyclone and the main result}

Before defining the property we call 2-additive balanced cyclone, let us give some basic notions of graph theory we need:

\footnotetext{
${ }^{1}$ We assume that multiple edges exist between two vertices in the graph. This type of graph is called multigraph.
} 
Definition 3.1. Let $x, y$ be two elements of $\mathcal{B}$.

1. Let $R$ be a binary relation on $\mathcal{B}$. A sequence $x_{1}, x_{2}, \cdots, x_{p}$ of elements of $\mathcal{B}$ is a path of $R$ from $x$ to $y$ if $x=x_{1} R x_{2} R \cdots R x_{p-1} R x_{p}=y$, and the sequence $x_{1}, x_{2}, \cdots, x_{p}$ contains at least two distinct elements.

- A path of $R$ from $x$ to $x$ is called a cycle of $R$.

- A path of $R$ from $x$ to $x$ is called an elementary cycle of $R$ if no element of the cycle is visited more than once (except $x$ ).

2. A path $\left\{x_{1}, x_{2}, \ldots, x_{p}\right\}$ of $\mathcal{R}$ is said to be a strict path from $x$ to $y$ if there exists $i$ in $\{1, \ldots, p-1\}$ such that $x_{i} \mathcal{P}^{\prime} x_{i+1}$. A strict path of $\mathcal{R}$ from $x$ to $x$ is called a strict cycle of $\mathcal{R}$.

Given a cycle $C$ of $\mathcal{R}$ and a binary relation $R$ in $\left\{P_{k}, P_{k}^{-1}, M, M_{A}, M_{A}^{-1}\right\}$, we denote by:

- $C \cap R$ the usual intersection between the cycle $C$ and $R$;

- $(x, y)_{R}$ the oriented edge from $x$ to $y$ belonging to $R$;

- $\{(x, y)\}$ the set of all edges of $\mathcal{R}$ between $x$ and $y$.

Remark 2. Since multiple edges are allowed between two vertices, we have in Figure 1: for the cycle $C$ of $\mathcal{R}$ defined by $a_{23} P_{1} a_{2} M_{\{1,2,4\}}{ }^{-1} a_{0} M_{\{1,3,4\}} a_{1} P_{2} a_{23}$, $\left|C \cap\left(a_{23}, a_{2}\right)_{M}\right|=0$ and $\left|C \cap\left\{\left(a_{23}, a_{2}\right)\right\}\right|=1$.

Definition 3.2. Let $m \in \mathbb{N} \backslash\{0\}$.

1. A m-cyclone of $\mathcal{R}$ is a nonempty union of at most $m$ cycles of $\mathcal{R}$. Thus a m-cyclone is obtained by taking elements in $\mathcal{R}$ that altogether can be partitioned into $m$ cycles.

2. A $m$-cyclone of $\mathcal{R}$ is said to be strict if it contains a strict cycle of $\mathcal{R}$.

3. A $m$-cyclone $\mathcal{C}$ of $\mathcal{R}$ is said to be 2 -additive balanced if it satisfies the two following conditions:

$$
\begin{gathered}
\forall k \in\{1,2, \ldots, q-1\},\left|\mathcal{C} \cap P_{k}^{-1}\right| \leq\left|\mathcal{C} \cap P_{k+1}\right| ; \\
\forall i, j \in N, i \neq j, \sum_{\substack{\{i, j\} \\
|A| \geq A}} \frac{1}{|A|-2}\left|\mathcal{C} \cap\left(a_{0}, a_{i}\right)_{M_{A}}\right| \leq\left|\mathcal{C} \cap\left(a_{i j}, a_{j}\right)_{M}\right| .
\end{gathered}
$$

The notion of balanced $m$-cyclone was first used by Doignon in [12] for the characterization of the representation of multiple semiorders by thresholds. The same notion and result is also presented in [32]. We have adapted this characterization in our context by introducing the term of 2-additive since we work with the 2-additive Choquet integral. Hence, we give below the main result of the paper, which is a theorem of characterization of consistent cardinal information $\left\{P, I, P_{1}, \ldots, P_{q}\right\}$ representable by a 2 -additive Choquet integral: 
Theorem 1. A cardinal information $\left\{P, I, P_{1}, \ldots, P_{q}\right\}$ is representable by a 2-additive Choquet integral on $\mathcal{B}$ if and only if no strict $(q+n(n-1))$-cyclone of $\mathcal{R}$ is 2-additive balanced.

This result shows that the only inconsistencies which can occur in the representation of a cardinal information are strict 2-additive balanced cyclone. The presence of cyclones in an inconsistent cardinal information is not surprising if we look at the characterizations of families of relations modeling preferences under incomplete information made by Mousset [27, 28]. She proved that, in the context of MACBETH methodology, the cardinal information corresponds to the family CHSF (Family of Semiorders with Homogeneity Constant thresholds) which is a subfamily of interval orders. She proposed also in [27] a characterization of the family CHSF by using the notion of "unordered pairs" which are equivalent to cyclone [29].

\section{Proofs}

We give here a proof of Theorem 1 in the same spirit of the proof of theorem of representation of multiple semiorders in $[12,32]$.

\subsection{Multigraph}

A weighted multigraph $G=(V, E, w)$ is defined as an oriented graph where $V$ is a finite set of vertices, $E$ is a family of couple of vertices called edges (with repetitions of the same couple allowed), and $w$ a weight mapping from $E$ to the real numbers. A cycle $C$ of $G$ is a finite sequence of edges having the form $\left(x_{1}, x_{2}\right)\left(x_{2}, x_{3}\right) \cdots\left(x_{p-1}, x_{p}\right)\left(x_{p}, x_{1}\right)$ with $p>1$. The weight of $C$ is defined as the sum of the weights of its edges.

Theorem 2. For any weighted multigraph $G=(V, E, w)$, the following two conditions are equivalent:

1. There exists $f: V \rightarrow \mathbb{R}$ such that $\forall(x, y) \in E$,

$$
f(x) \geq f(y)+w(x, y) ;
$$

2. No cycle of $G$ has a strictly positive weight.

Proof. See [36].

For a cycle $C$ of $\mathcal{R}$, we use the following notations:

1. $\forall k \in\{1, \cdots, q-1\}$,

$$
b_{k}(C)=\left|C \cap P_{k}^{-1}\right|-\left|C \cap P_{k+1}\right|
$$

2. $\forall i, j \in N, i \neq j$,

$$
b_{i j}^{i}(C)=\left(\sum_{\substack{\{i, j\} \subseteq A \subseteq N \\|A| \geq 3}} \frac{1}{|A|-2}\left|C \cap\left(a_{0}, a_{i}\right)_{M_{A}}\right|\right)-\left|C \cap\left(a_{i j}, a_{j}\right)_{M}\right|
$$


Lemma 5. Let be $\sigma_{1}, \sigma_{2}, \ldots, \sigma_{q-1}$ real numbers and $\sigma_{i j}^{i}$ nonnegative real numbers with $i, j \in N, i \neq j$.

There exists $f: \mathcal{B} \rightarrow \mathbb{R}_{+}$such that

$$
\begin{aligned}
& \forall(x, y) \in I: f(x)=f(y), \\
& \forall k \in\{1, \ldots, q\}, \forall(x, y) \in P_{k}: \sigma_{k-1}<f(x)-f(y), \quad\left(\sigma_{0}:=0\right) \\
& \forall k \in\{1, \ldots, q-1\}, \forall(x, y) \in P_{k}: f(x)-f(y)<\sigma_{k}, \\
& f\left(a_{0}\right)=0, \\
& \forall i, j \in N, i \neq j: f\left(a_{i j}\right)-f\left(a_{j}\right) \geq \sigma_{i j}^{i}, \\
& \forall A \subseteq N,|A| \geq 3, \forall i \in A: 0 \leq f\left(a_{i}\right) \leq \frac{1}{|A|-2} \sum_{j \in A \backslash\{i\}} \sigma_{i j}^{i} .
\end{aligned}
$$

if and only if the following two conditions are satisfied:

1. Any cycle ${ }^{2} C$ of $\mathcal{R}$ satisfies

$$
\sum_{k=1}^{q-1} b_{k}(C) \sigma_{k}+\sum_{i \in N} \sum_{j \in N \backslash i} b_{i j}^{i}(C) \sigma_{i j}^{i} \geq 0
$$

2. Any strict cycle $C$ of $\mathcal{R}$ satisfies

$$
\sum_{k=1}^{q-1} b_{k}(C) \sigma_{k}+\sum_{i \in N} \sum_{j \in N \backslash i} b_{i j}^{i}(C) \sigma_{i j}^{i}>0
$$

Proof.

- $(\Rightarrow)$ Let $C$ be a cycle of $\mathcal{R}$. For each element $(x, y)$ of $C$, we have:

$* f(x)-f(y)=0$ if $(x, y) \in I$;

$* f(x)-f(y)>\sigma_{k-1}$ if $(x, y) \in P_{k}$;

$* f(x)-f(y)>-\sigma_{k}$ if $(x, y) \in P_{k}^{-1}$;

$* f(x)-f(y) \geq \sigma_{i j}^{i}$ if $(x, y)=\left(a_{i j}, a_{j}\right) \in M$;

* $f(x)-f(y) \geq 0$ if $(x, y)=\left(a_{i}, a_{0}\right) \in M_{A}^{-1}, A \subseteq N,|A| \geq 3$;

* $f(x)-f(y) \geq-\left(\frac{1}{|A|-2} \sum_{j \in A \backslash\{i\}} \sigma_{i j}^{i}\right)$ if $(x, y)=\left(a_{0}, a_{i}\right) \in M_{A}, A \subseteq$ $N,|A| \geq 3$.

Since $C$ is a cycle of $\mathcal{R}$, we have $\sum_{(x, y) \in C}[f(x)-f(y)]=0$. Hence

${ }^{2}$ elementary or not 
$0 \geq \sum_{k=1}^{q}\left|C \cap P_{k}\right| \sigma_{k-1}-\sum_{k=1}^{q-1}\left|C \cap P_{k}^{-1}\right| \sigma_{k}+\sum_{i \in N} \sum_{j \in N \backslash\{i\}}\left|C \cap\left(a_{i j}, a_{j}\right)_{M}\right| \sigma_{i j}^{i}-$ $\sum_{|A| \geq 3} \sum_{i \in A \subseteq N}\left|C \cap\left(a_{0}, a_{i}\right)_{M_{A}}\right|\left(\frac{1}{|A|-2} \sum_{j \in A \backslash\{i\}} \sigma_{i j}^{i}\right)$ (this inequality is strict if $C$ is strict).

We note that:

$$
\begin{aligned}
\text { 1. } & \sum_{k=1}^{q}\left|C \cap P_{k}\right| \sigma_{k-1}-\sum_{k=1}^{q-1}\left|C \cap P_{k}^{-1}\right| \sigma_{k} \\
= & \left.\sum_{k=1}^{q-1}\left(\left|C \cap P_{k+1}\right|-\left|C \cap P_{k}^{-1}\right|\right) \sigma_{k} \text { (knowing that } \sigma_{0}=0\right) \\
= & \sum_{k=1}^{q-1}\left(-b_{k}(C)\right) \sigma_{k} . \\
\text { 2. } & \sum_{i \in N} \sum_{j \in N \backslash\{i\}}\left|C \cap\left(a_{i j}, a_{j}\right)_{M}\right| \sigma_{i j}^{i}-\sum_{|A| \geq 3} \sum_{i \in A \subseteq N}\left|C \cap\left(a_{0}, a_{i}\right)_{M_{A}}\right|\left(\frac{1}{|A|-2} \sum_{j \in A \backslash\{i\}} \sigma_{i j}^{i}\right) \\
& =\sum_{i \in N} \sum_{j \in N \backslash\{i\}}\left(\left|C \cap\left(a_{i j}, a_{j}\right)_{M}\right|-\left[\sum_{\substack{\{i, j\} \subseteq A \subseteq N \\
|A| \backslash 3}} \frac{1}{|A|-2}\left|C \cap\left(a_{0}, a_{i}\right)_{M_{A}}\right|\right] \sigma_{i j}^{i}\right. \\
& =\sum_{i \in N} \sum_{j \in N \backslash\{i\}}\left(-b_{i j}^{i}(C)\right) \sigma_{i j}^{i}
\end{aligned}
$$

Therefore

$$
\sum_{k=1}^{q-1} b_{k}(C) \sigma_{k}+\sum_{i \in N} \sum_{j \in N \backslash i} b_{i j}^{i}(C) \sigma_{i j}^{i} \geq 0,
$$

this inequality being strict if $C$ is strict. Hence (44) and (45) are satisfied.

- $(\Leftarrow)$ Conversely we suppose that $(44)$ et $(45)$ are satisfied. Let $\mathcal{C}\left(\mathcal{P}^{\prime}\right)$ the set of all strict cycles of $\mathcal{R}$. The set $\mathcal{C}\left(\mathcal{P}^{\prime}\right)$ is always nonempty and its cardinality is finite. Hence there exist an $\epsilon>0$ such that for each strict cycle $C$ of $\mathcal{R}$, we have

$$
\sum_{k=1}^{q-1}\left(\left|C \cap P_{k}^{-1}\right|-\left|C \cap P_{k+1}\right|\right) \sigma_{k}+\sum_{i \in N} \sum_{j \in N \backslash i} b_{i j}^{i} \sigma_{i j}^{i}-\epsilon \sum_{k=1}^{q-1}\left(\left|C \cap P_{k+1}\right|+\left|C \cap P_{k}^{-1}\right|\right) \geq 0 .
$$

To see this, it is sufficient to take

$$
\epsilon=\min _{C \in \mathcal{C}\left(\mathcal{P}^{\prime}\right)} \frac{\sum_{k=1}^{q-1}\left(\left|C \cap P_{k}^{-1}\right|-\left|C \cap P_{k+1}\right|\right) \sigma_{k}+\sum_{i \in N} \sum_{j \in N \backslash i} b_{i j}^{i}(C) \sigma_{i j}^{i}}{\sum_{k=1}^{q-1}\left(\left|C \cap P_{k+1}\right|+\left|C \cap P_{k}^{-1}\right|\right)} .
$$


Therefore, we get the following equation:

$$
\sum_{k=1}^{q}\left|C \cap P_{k}\right|\left(\sigma_{k-1}+\epsilon\right)+\sum_{k=1}^{q-1}\left|C \cap P_{k}^{-1}\right|\left(-\sigma_{k}+\epsilon\right)+\sum_{i \in N} \sum_{j \in N \backslash i}\left(-b_{i j}^{i}(C)\right) \sigma_{i j}^{i} \leq 0 .
$$

Since equation (44) is satisfied, we get for any nonstrict cycle $C$ of $\mathcal{R}$ the following equation (knowing that in this case $\left|C \cap P_{k}\right|=\left|C \cap P_{k}^{-1}\right|=0$ ):

$$
\begin{aligned}
& \sum_{i \in N} \sum_{j \in N \backslash\{i\}}\left|C \cap\left(a_{i j}, a_{j}\right)_{M}\right| \sigma_{i j}^{i} \\
& -\sum_{|A| \geq 3} \sum_{i \in A \subseteq N}\left|C \cap\left(a_{0}, a_{i}\right)_{M_{A}}\right|\left(\frac{1}{|A|-2} \sum_{j \in A \backslash\{i\}} \sigma_{i j}^{i}\right) \leq 0
\end{aligned}
$$

Let us consider the oriented multigraph $G=(V, E, w)$ where $V=\mathcal{B}$, $E=\mathcal{R}$ and $w$ are defined as follows: $\forall(x, y) \in E$,

$$
w(x, y)= \begin{cases}0 & \text { if }(x, y) \in I \\ \epsilon & \text { if }(x, y) \in P_{1} \\ \sigma_{k-1}+\epsilon & \text { if }(x, y) \in P_{k}, k \in\{2, \ldots, q\} \\ -\sigma_{k}+\epsilon & \text { if }(x, y) \in P_{k}^{-1}, k \in\{1, \ldots, q-1\} \\ \sigma_{i j}^{i} & \text { if }(x, y)=\left(a_{i j}, a_{j}\right) \in M \\ 0 & \text { if }(x, y)=\left(a_{i}, a_{0}\right) \in M_{A}^{-1}, A \subseteq N,|A| \geq 3 \\ -\left(\frac{1}{|A|-2} \sum_{j \in A \backslash\{i\}} \sigma_{i j}^{i}\right) & \text { if }(x, y)=\left(a_{0}, a_{i}\right) \in M_{A}, A \subseteq N,|A| \geq 3 .\end{cases}
$$

Equations (46) and (47) show that any cycle of $G$ has a negative weight. Using Theorem 2, there exists $g: \mathcal{B} \rightarrow \mathbb{R}$ such that $\forall(x, y) \in E$,

$$
g(x) \geq g(y)+w((x, y)) .
$$

Therefore we have

$$
\forall(x, y) \in I: g(x)=g(y)
$$

$$
\forall k \in\{1, \ldots, q\}, \forall(x, y) \in P_{k}: \sigma_{k-1}<g(x)-g(y),\left(\sigma_{0}=0\right)
$$

$$
\forall k \in\{1, \ldots, q-1\}, \forall(x, y) \in P_{k}: g(x)-g(y)<\sigma_{k}
$$

$\forall i, j \in N, i \neq j, \forall\left(a_{i j}, a_{j}\right) \in M: g\left(a_{i j}\right)-g\left(a_{j}\right) \geq \sigma_{i j}^{i}$,

$\forall A \subseteq N,|A| \geq 3, \forall\left(a_{0}, a_{i}\right) \in M_{A}: 0 \leq g\left(a_{i}\right)-g\left(a_{0}\right) \leq \frac{1}{|A|-2} \sum_{j \in A \backslash\{i\}} \sigma_{i j}^{i}$. 
If we consider the function $f: \mathcal{B} \rightarrow \mathbb{R}_{+}$defined by:

$$
\left\{\begin{array}{l}
f\left(a_{0}\right)=0 ; \\
f(x)=g(x)-g\left(a_{0}\right), \forall x \in \mathcal{B} \backslash\left\{a_{0}\right\}
\end{array}\right.
$$

then $f$ satisfies the equations (38), (39), (40), (41),(42) and (43).

\subsection{The Helly's theorem, Farkas Lemma and the proof of Theorem 1}

In this section, we need the Helly's theorem and the following theorem which is a corollary of Mangasarian's [22] version of the Farkas Lemma

Theorem 3. Let be $A \in \mathbb{R}^{p \times m}, B \in \mathbb{R}^{r \times m}$ and $C \in \mathbb{R}^{q \times m}$ three matrices, with $m, p, q, r \in \mathbb{N}, A \neq 0$. Then either

1. There exists $X \in \mathbb{R}^{m}$ such that $\left\{\begin{array}{l}\text { A.X }>0 \\ B . X=0 \\ \text { C.X } \geq 0\end{array}\right.$

or

2. There exists $Y \in \mathbb{R}^{p}, Z \in \mathbb{R}^{r}$ and $U \in \mathbb{R}^{q}$ such that $\left\{\begin{array}{l}{ }^{t} A . Y+{ }^{t} B . Z+{ }^{t} C . U=0 \\ Y \geq 0, Y \neq 0 \\ U \geq 0 .\end{array}\right.$

Proof. See [22, 35].

Remark 3. If $A \in \mathbb{Z}^{p \times m}, B \in \mathbb{Z}^{r \times m}$ and $C \in \mathbb{Z}^{q \times m}$ then Theorem 3 can be rewritten with $Y \in \mathbb{Z}^{p}, Z \in \mathbb{Z}^{r}$ and $U \in \mathbb{Z}^{q}$.

Theorem 4. Let $C_{1}, \ldots, C_{m}, m>n$ be convex sets in $n$ dimensional Euclidean space $E^{n}$. If each collection of $n+1$ of these sets have a point in common then there is a common point to all $C_{i}, i=1,2, \ldots, m$.

Equivalently the theorem states that if $\bigcap_{k=1}^{m} C_{i}=\emptyset$, then there exist $k+1$ (with $k \leq n$ ) sets $C_{i_{1}}, \ldots, C_{i_{k}}$ such that $C_{i_{1}} \cap \cdots \cap C_{i_{k}}=\emptyset$

Proof. See [33], Theorem 21.6, p.196.

\subsubsection{Proof of Theorem 1}

Let $\left\{P, I, P_{1}, \ldots, P_{q}\right\}$ a cardinal information on $\mathcal{B}$.

1. $(\Rightarrow)$ Suppose that $\left\{P, I, P_{1}, \ldots, P_{q}\right\}$ is representable by a 2 -additive Choquet integral on $\mathcal{B}$, i.e., there exist $f: \mathcal{B} \rightarrow \mathbb{R}_{+}$and $(q-1)$ real numbers $\sigma_{1}, \sigma_{2}, \ldots, \sigma_{q-1}$ and nonnegative numbers $\sigma_{i j}^{i}, i, j \in N, i \neq j$ such that the equations (30), (31), (32), (33) (34) and (35) are satisfied. Using Remark 1 the real numbers $\sigma_{1}, \sigma_{2}, \ldots, \sigma_{q-1}$ are also nonnegative. 
Let $\mathcal{C}$ be a strict $(q+n(n-1))$-cyclone of $\mathcal{R}$. Because $\mathcal{C}$ contains a strict cycle, by applying inequalities (44) and (45) of Lemma 5 to each cycle of $\mathcal{C}$ and summing up all these inequalities, we obtain:

$$
\begin{aligned}
& \sum_{k=1}^{q-1}\left(\left|\mathcal{C} \cap P_{k}^{-1}\right|-\left|\mathcal{C} \cap P_{k+1}\right|\right) \sigma_{k}+\sum_{i \in N} \sum_{j \in N \backslash\{i\}}\left[\left(\sum_{\substack{\{i, j\} \subseteq A \subseteq N \\
|A| \geq 3}} \frac{1}{|A|-2} \mid \mathcal{C} \cap\right.\right. \\
& \left.\left.\left(a_{0}, a_{i}\right)_{M_{A}} \mid\right)-\left|\mathcal{C} \cap\left(a_{i j}, a_{j}\right)_{M}\right|\right] \sigma_{i j}^{i}>0 .
\end{aligned}
$$

If $\mathcal{C}$ is 2 -additive balanced then we have the following contradiction:

$$
\begin{aligned}
& \sum_{k=1}^{q-1}\left(\left|\mathcal{C} \cap P_{k}^{-1}\right|-\left|\mathcal{C} \cap P_{k+1}\right|\right) \sigma_{k}+\sum_{i \in N} \sum_{j \in N \backslash\{i\}}\left[\left(\sum_{\substack{\{i, j\} \subseteq A \subseteq N \\
|A| \geq 3}} \frac{1}{|A|-2} \mid \mathcal{C} \cap\right.\right. \\
& \left.\left.\left(a_{0}, a_{i}\right)_{M_{A}} \mid\right)-\left|\mathcal{C} \cap\left(a_{i j}, a_{j}\right)_{M}\right|\right] \quad \sigma_{i j}^{i} \leq 0 .
\end{aligned}
$$

2. $(\Leftarrow)$ Suppose that no strict $(q+n(n-1))$-cyclone of $\mathcal{R}$ is 2 -additive balanced. According to Lemma 5, a sufficient condition for the representation of $\left\{P, I, P_{1}, \ldots, P_{q}\right\}$ by a 2 -additive Choquet integral is that there exist a common solution with real numbers $\sigma_{1}, \sigma_{2}, \ldots, \sigma_{q-1}$ and nonnegative real numbers $\sigma_{i j}^{i}$ with $i, j \in N, i \neq j$ to the system of inequalities (44) and (45), associated to the set of all nonempty cycles $C$ of $\mathcal{R}$. We suppose that $\mathcal{R}$ contains $r$ nonempty cycles $C_{1}, C_{2}, \ldots, C_{r}$. Let $I$ be the set defined by $I=\left\{i \in\{1, \ldots, r\}: C_{i}\right.$ is a strict cycle of $\left.\mathcal{R}\right\}$. We look for real values $\sigma_{1}, \sigma_{2}, \ldots, \sigma_{q-1}, \sigma_{i j}^{i}$ with $i, j \in N, i \neq j$ such that the following system is satisfied:

$$
\begin{cases}\sum_{k=1}^{q-1} b_{k}\left(C_{l}\right) \sigma_{k}+\sum_{i \in N} \sum_{j \in N \backslash i} b_{i j}^{i}\left(C_{l}\right) \sigma_{i j}^{i} \geq 0 & \forall l \in\{1, \ldots, r\} \backslash I \\ \sum_{k=1}^{q-1} b_{k}\left(C_{l}\right) \sigma_{k}+\sum_{i \in N} \sum_{j \in N \backslash i} b_{i j}^{i}\left(C_{l}\right) \sigma_{i j}^{i}>0 & \forall l \in I \\ \sigma_{i j}^{i} \geq 0 & \forall i, j \in N, i \neq j\end{cases}
$$

Each such inequality determines a half space in $\mathbb{R}^{q-1+n(n-1)}$, the space of $(q-1+n(n-1))$-tuples $\left(\sigma_{1}, \ldots, \sigma_{q-1}, \sigma_{12}^{1}, \sigma_{12}^{2}, \ldots, \sigma_{(n-1) n}^{n-1}, \sigma_{(n-1) n}^{n}\right)$. By Helly's theorem (see Theorem 4), the intersection of all half spaces determined by the above inequalities is nonempty if and only if any subsystem of $q+n(n-1)$ inequalities has a solution. Let us consider a subsystem $S^{\prime}$ of $q+n(n-1)$ inequalities of the system (53).

(a) If $S^{\prime}$ does not contain an inequality coming from a cycle $C_{l}$ such that $l \in I$, then $\sigma=\left\{\begin{array}{ll}\sigma_{k}=1 & \forall k \in\{1, \ldots, q-1\}, \\ \sigma_{i j}^{i}=0 & \forall i, j \in N, i \neq j,\end{array} \quad\right.$ is a solution of $S^{\prime}$ 
since $\forall h \in\{1, \ldots, r\} \backslash I, \forall k \in\{1, \ldots, q-1\},\left|C_{h} \cap P_{k}\right|=\left|C_{h} \cap P_{k}^{-1}\right|=$ 0 .

(b) Without loss of the generality, we suppose that $S^{\prime}$ is composed by $m^{\prime}$ inequalities coming from the cycles $C_{l_{1}}, C_{l_{2}}, \ldots, C_{l_{m^{\prime}}}$ with $\left\{l_{1}, \ldots, l_{m^{\prime}}\right\} \subseteq$ $I$, and $m^{\prime \prime}$ inequalities coming from the cycles $C_{l_{m^{\prime}+1}}, C_{l_{m^{\prime}+2}}, \ldots, C_{l_{m^{\prime}+m^{\prime \prime}}}$, with $\left\{l_{m^{\prime}+1}, \ldots, l_{m^{\prime}+m^{\prime \prime}}\right\} \subseteq\{1, \ldots, r\} \backslash I$. Knowing that $b_{k}\left(C_{l_{h}}\right)=0$ for $h=m^{\prime}+1, \ldots, m^{\prime}+m^{\prime \prime}$, the system $S^{\prime}$ is given as follows:

$$
\left\{\begin{array}{l}
\sum_{k=1}^{q-1} b_{k}\left(C_{l_{1}}\right) \sigma_{k}+\sum_{i \in N} \sum_{j \in N \backslash i} b_{i j}^{i}\left(C_{l_{1}}\right) \sigma_{i j}^{i}>0 \\
\sum_{k=1}^{q-1} b_{k}\left(C_{l_{2}}\right) \sigma_{k}+\sum_{i \in N} \sum_{j \in N \backslash i} b_{i j}^{i}\left(C_{l_{2}}\right) \sigma_{i j}^{i}>0 \\
\vdots \\
\sum_{k=1}^{q-1} b_{k}\left(C_{l_{m^{\prime}}}\right) \sigma_{k}+\sum_{i \in N} \sum_{j \in N \backslash i} b_{i j}^{i}\left(C_{l_{m^{\prime}}}\right) \sigma_{i j}^{i}>0 \\
\sum_{i \in N} \sum_{j \in N \backslash i} b_{i j}^{i}\left(C_{l_{m^{\prime}+1}}\right) \sigma_{i j}^{i} \geq 0 \\
\sum_{i \in N} \sum_{j \in N \backslash i} b_{i j}^{i}\left(C_{l_{m^{\prime}+2}}\right) \sigma_{i j}^{i} \geq 0 \\
\vdots \\
\sum_{i \in N} \sum_{j \in N \backslash i} b_{i j}^{i}\left(C_{l_{m^{\prime}+m^{\prime \prime}}}\right) \sigma_{i j}^{i} \geq 0 \\
\sigma_{i j}^{i} \geq 0, \forall i, j \in N\left(\text { for the other } q+n(n-1)-\left(m^{\prime}+m^{\prime \prime}\right)\right. \\
\text { nonnegativity inequalities })
\end{array}\right.
$$

If $S^{\prime}$ has no solution, then using Theorem 3 and Remark 3 , there exists nonnegative integers $\alpha_{1}, \ldots, \alpha_{m^{\prime}}, \alpha_{m^{\prime}+1}, \ldots, \alpha_{m^{\prime}+m^{\prime \prime}}$ with $\alpha_{t}>0$ for some $t \in\left\{1, \ldots, m^{\prime}\right\}$, and $\beta_{i j}^{i}$ for all $i, j \in N$ such that the following system is satisfied:

$$
\begin{cases}\sum_{h=1}^{m^{\prime}} \alpha_{h} b_{k}\left(C_{l_{h}}\right)=0 & \forall k=1,2, \ldots, q-1 \\ \sum_{h=1}^{m^{\prime}+m^{\prime \prime}} \alpha_{h} b_{i j}^{i}\left(C_{l_{h}}\right)+\beta_{i j}^{i}=0 & \forall i, j \in N, i \neq j\end{cases}
$$

Hence we have

$$
\begin{cases}\sum_{h=1}^{m^{\prime}} \alpha_{h} b_{k}\left(C_{l_{h}}\right)=0 & \forall k=1,2, \ldots, q-1 \\ \sum_{h=1}^{m^{\prime}+m^{\prime \prime}} \alpha_{h} b_{i j}^{i}\left(C_{l_{h}}\right) \leq 0 & \forall i, j \in N, i \neq j\end{cases}
$$


i.e.

$$
\left\{\begin{array}{l}
\sum_{h=1}^{m^{\prime}} \alpha_{h}\left|C_{l_{h}} \cap P_{k}^{-1}\right|=\sum_{h=1}^{m^{\prime}} \alpha_{h}\left|C_{l_{h}} \cap P_{k+1}\right| \forall k=1,2, \ldots, q-1 \\
\sum_{h=1}^{m^{\prime}+m^{\prime \prime}} \alpha_{h}\left(\sum_{\substack{\{i, j\} \subseteq A \subseteq N \\
|A| \geq 3}} \frac{1}{|A|-2}\left|C_{l_{h}} \cap\left(a_{0}, a_{i}\right)_{M_{A}}\right|\right) \\
\leq \sum_{h=1}^{m^{\prime}+m^{\prime \prime}} \alpha_{h}\left|C_{l_{h}} \cap\left(a_{i j}, a_{j}\right)_{M}\right| \quad \forall i, j \in N, i \neq j .
\end{array}\right.
$$

Let us consider the strict $(q+n(n-1))$-cyclone $\mathcal{C}$ composed of the cycles $C_{l_{h}}^{\prime}, h=1, \ldots, m^{\prime}+m^{\prime \prime}$ where $C_{l_{h}}^{\prime}$ consists in passing $\alpha_{h}$ times through the cycle $C_{l_{h}}$. The resulting $(q+n(n-1))$-cyclone $\mathcal{C}$ uses $\sum_{h=1}^{m^{\prime}} \alpha_{h}\left|C_{l_{h}} \cap P_{k}^{-1}\right|$ edges from $P_{k}^{-1}, \sum_{h=1}^{m^{\prime}} \alpha_{h}\left|C_{l_{h}} \cap P_{k+1}\right|$ edges from $P_{k+1}, \sum_{h=1}^{m^{\prime}+m^{\prime \prime}} \alpha_{h}\left|C_{l_{h}} \cap\left(a_{0}, a_{i}\right)_{M_{A}}\right|$ edges from $M_{A} \cap\left\{\left(a_{0}, a_{i}\right)\right\}$ and $\sum_{h=1}^{m^{\prime}+m^{\prime \prime}} \alpha_{h}\left|C_{l_{h}} \cap\left(a_{i j}, a_{j}\right)_{M}\right|$ edges from $M \cap\left\{\left(a_{i j}, a_{j}\right)\right\}$. Therefore, we deduce from the system (57) the following system:

$$
\left\{\begin{array}{l}
\sum_{h=1}^{m^{\prime}}\left|C_{l_{h}}^{\prime} \cap P_{k}^{-1}\right|=\sum_{h=1}^{m^{\prime}}\left|C_{l_{h}}^{\prime} \cap P_{k+1}\right| \quad \forall k=1,2, \ldots, q-1 \\
\sum_{h=1}^{m^{\prime}+m^{\prime \prime}}\left(\sum_{\substack{\{i, j\} \subseteq A \subseteq N \\
|A| \geq 3}} \frac{1}{|A|-2}\left|C_{l_{h}}^{\prime} \cap\left(a_{0}, a_{i}\right)_{M_{A}}\right|\right) \\
\leq \sum_{h=1}^{m^{\prime}+m^{\prime \prime}}\left|C_{l_{h}}^{\prime} \cap\left(a_{i j}, a_{j}\right)_{M}\right| \quad \forall i, j \in N, i \neq j
\end{array}\right.
$$

i.e.

$$
\left\{\begin{array}{l}
\sum_{\substack{\left|\mathcal{C} \cap P_{k}^{-1}\right|=\left|\mathcal{C} \cap P_{k+1}\right| \\
\{i, j \backslash A \subseteq N \\
\forall i, j \in N, i \neq j}} \frac{1}{|A|-2}\left|\mathcal{C} \cap M_{A} \cap\left\{\left(a_{0}, a_{i}\right)\right\}\right| \leq\left|\mathcal{C} \cap M \cap\left\{\left(a_{i j}, a_{j}\right)\right\}\right| \\
\forall i, j \in N, i \neq j
\end{array}\right.
$$

that means $\mathcal{C}$ is a 2 -additive balanced strict $(q+n(n-1))$-cyclone, a contradiction with the hypothesis. 


\section{An alternative to the 2-additive balanced cyclones?}

In practice, cyclones are not easy to detect because they allow multiple edges between two vertices. This problem occurs when we want to explain to a DM how to manage the inconsistencies by giving him some recommendations in order to have consistent information. Therefore we can ask if it is possible to find simple necessary and sufficient conditions equivalent to the 2 -additive balanced cyclone condition? This question still an open and difficult problem to solve. When a cardinal information is inconsistent, we propose to test if this information satisfy some simple necessary conditions such as MOPI ${ }^{3}$ and MOPIC conditions presented below. If these conditions are satisfied, linear programming technics such as the determination of an irreducible inconsistent system (ISS) [2, 9] can be applied in order to deal with inconsistencies. Before defining the MOPIC properties, let us introduce the following notations and definitions:

\section{Definition 5.1.}

1. We introduce the relation $M^{\prime}$ defined such that for $(x, y) \in\left\{\left(a_{i}, a_{0}\right), i \in N\right\} \cup\left\{\left(a_{i j}, a_{i}\right), i, j \in N, i \neq j\right\}$,

$$
x M^{\prime} y \text { if } \operatorname{not}(x(P \cup I) y) \text {. }
$$

2. $i$ - A path of $\left(P \cup I \cup M^{\prime}\right)$ from $x$ to $y$ is denoted by $x T C y$. We use also the notation $x T C_{P_{l}} y$ if this path contains an element of $P_{l}$;

ii- We write $x \sim y$ if there exists a cycle of $\left(I \cup M^{\prime}\right)$ containing $x$ and $y$.

3. For all $i, j \in N$, the notation $i \vee j$ denotes one of the two criteria $i$ or $j$.

Definition 5.2. A cardinal information $\left\{P, I, P_{1}, \ldots, P_{q}\right\}$ on $\mathcal{B}$ satisfies the property

1. MOPIC-1 if for all $i, j \in N, i \neq j$,

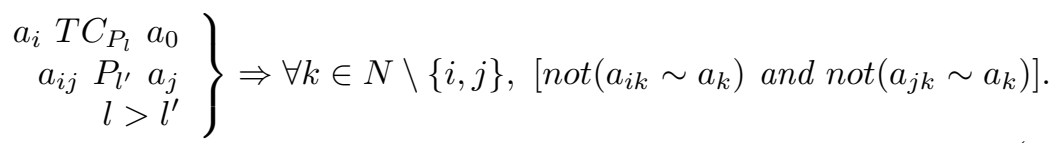

2. MOPIC-2 if for all distinct $i, j, k \in N$,

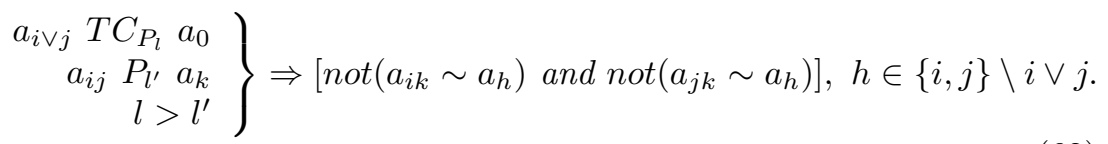

\footnotetext{
${ }^{3}$ MOnotonicity of Preferential Information. The MOPI condition defined and used in [24] as necessary and sufficient conditions to represent an ordinal information (a cardinal information without intensity) by a 2 -additive Choquet integral.
} 


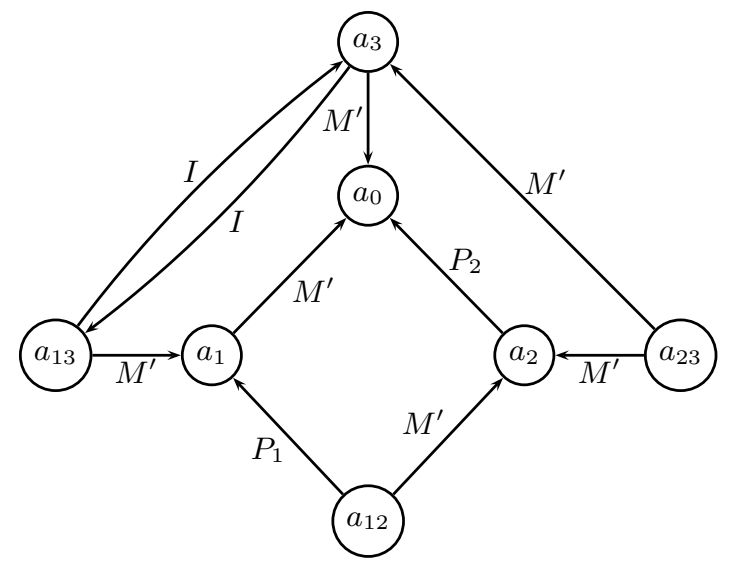

Figure 2: MOPIC-1 not satisfied. MOPIC-2 and MOPIC-3 satisfied

3. MOPIC-3 if for all distinct $i, j, k \in N$,

$\left.\begin{array}{rrr}a_{k} & T C_{P_{l}} & a_{0} \\ a_{i j} & P_{l^{\prime}} & a_{i \vee j} \\ & l>l^{\prime}\end{array}\right\} \Rightarrow\left[\operatorname{not}\left(a_{i k} \sim a_{h}\right)\right.$ and $\left.\operatorname{not}\left(a_{j k} \sim a_{h}\right)\right], h \in\{i, j\} \backslash i \vee j$.

The following example shows that the three MOPIC conditions are independent.

\section{Example 2.}

1. $N=\{1,2,3\}, I=\left\{\left(a_{13}, a_{3}\right)\right\}, P_{1}=\left\{\left(a_{12}, a_{1}\right)\right\}$ and $P_{2}=\left\{\left(a_{2}, a_{0}\right)\right\}$.

The property MOPIC-1 is not satisfied because we have $a_{2} P_{2} a_{0}, a_{12} P_{1} a_{1}$ and $a_{13} \sim a_{3}$. In this example, MOPIC-2 and MOPI-3 are satisfied (see Figure 2).

2. $N=\{1,2,3\}, I=\left\{\left(a_{23}, a_{1}\right)\right\}, P_{1}=\left\{\left(a_{12}, a_{3}\right)\right\}$ and $P_{2}=\left\{\left(a_{2}, a_{0}\right)\right\}$. MOPIC-2 is not satisfied because $a_{2} P_{2} a_{0}, a_{12} P_{1} a_{3}$ and $a_{23} \sim a_{1}$. On the other hand, MOPIC-1 and MOPI-3 are satisfied (see Figure 3).

3. $N=\{1,2,3\}, I=\left\{\left(a_{12}, a_{3}\right)\right\}, P_{1}=\left\{\left(a_{13}, a_{1}\right)\right\}$ and $P_{2}=\left\{\left(a_{2}, a_{0}\right)\right\}$.

The properties MOPIC-1 and MOPI-2 are satisfied but not MOPIC-3 because we have $a_{2} P_{2} a_{0}, a_{13} P_{1} a_{1}$ and $a_{12} \sim a_{3}$ (see Figure 4 ).

We proved in Proposition 1 that MOPIC conditions are necessary, but they are not sufficient for the representation of a cardinal information by a 2-additive Choquet integral. Indeed, if we take the following example for four criteria, $I=$ $\left\{\left(a_{12}, a_{3}\right),\left(a_{14}, a_{2}\right)\right\}, P_{1}=\left\{\left(a_{13}, a_{1}\right)\right\}, P_{2}=\left\{\left(a_{4}, a_{0}\right)\right\}$, it is easy to see that the properties MOPIC-1, MOPIC-2 and MOPIC-3 are satisfied, but $\left\{I, P_{1}, P_{2}\right\}$ is not representable by a 2 -additive Choquet integral $C_{\mu}$ because the monotonicity constraint $\mu_{12}+\mu_{13}+\mu_{14} \geq \mu_{1}+\mu_{1}+\mu_{2}+\mu_{3}+\mu_{4}$ is not satisfied. 


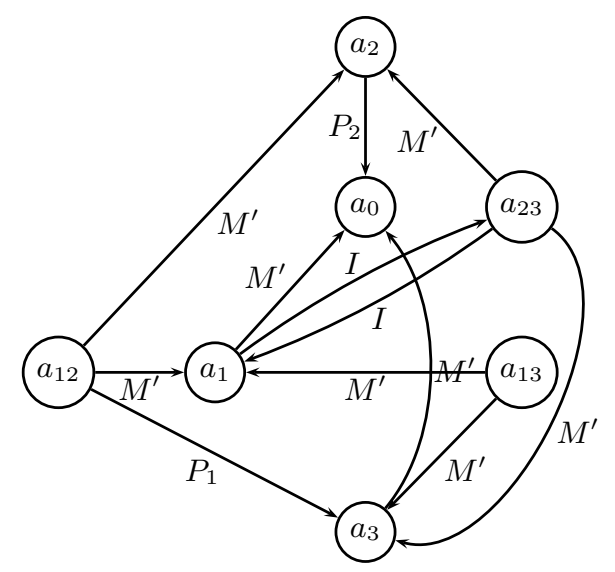

Figure 3: MOPIC-2 not satisfied. MOPIC-1 and MOPIC-3 satisfied

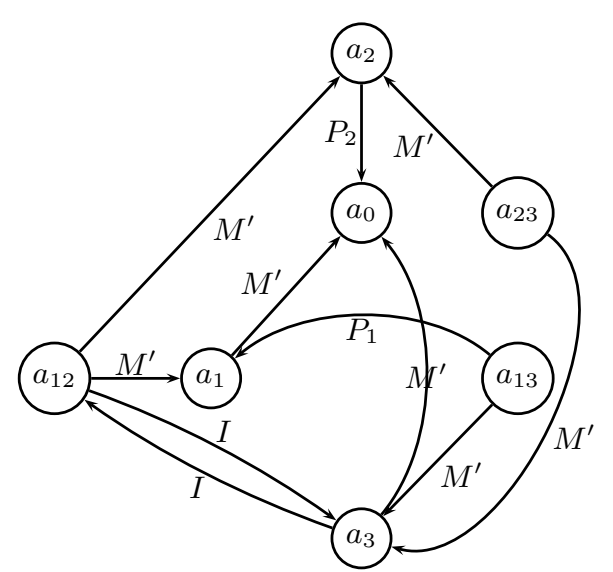

Figure 4: MOPIC-3 not satisfied. MOPIC-1 and MOPIC-2 satisfied 
Proposition 1. If a cardinal information $\left\{P, I, P_{1}, \ldots, P_{q}\right\}$ on $\mathcal{B}$ is representable by a 2-additive Choquet integral, then the properties MOPIC-1, MOPIC2 and MOPIC-3 are satisfied.

Proof. Suppose $\left\{P, I, P_{1}, \ldots, P_{q}\right\}$ representable by a 2 -additive Choquet integral $C_{\mu}$.

1. We prove first that for all $x, y, z, w \in \mathcal{B}$,

$$
\left\{\begin{array}{l}
x P_{l} y \\
z T C_{P_{h}} w \Rightarrow C_{\mu}(U(z))-C_{\mu}(U(w))>C_{\mu}(U(x))-C_{\mu}(U(y)) . \\
l<h
\end{array}\right.
$$

If $z T C_{P_{h}} w$ then there exist $z_{1}, z_{2} \in \mathcal{B}$ such that $z T C z_{1} P_{h} z_{2} T C w$.

Hence we have $C_{\mu}(U(z))-C_{\mu}(U(w)) \geq C_{\mu}\left(U\left(z_{1}\right)\right)-C_{\mu}\left(U\left(z_{2}\right)\right)$.

Since $l<h \Rightarrow C_{\mu}\left(U\left(z_{1}\right)\right)-C_{\mu}\left(U\left(z_{2}\right)\right)>C_{\mu}(U(x))-C_{\mu}(U(y)$, we have $C_{\mu}(U(z))-C_{\mu}(U(w))>C_{\mu}(U(x))-C_{\mu}(U(y))$.

2. (a) MOPIC-1:

Let $i, j \in N$ such that $\left\{\begin{array}{l}a_{i} T C_{P_{l}} a_{0} \\ a_{i j} P_{l^{\prime}} a_{j} \\ l>l^{\prime}\end{array}\right.$. By using the first point of the proposition, we have

$$
\mu_{i}>\mu_{i j}-\mu_{j}
$$

Suppose that there exists $k \in N \backslash\{i, j\}$ such that $a_{i k} \sim a_{k}$, then we have

$$
\mu_{i k}=\mu_{k}
$$

Combining (65) and (66), we obtain

$$
\mu_{i}+\mu_{j}+\mu_{k}>\mu_{i j}+\mu_{i k}
$$

A contradiction with the monotonicity constraint $\mu_{i j}+\mu_{i k} \geq \mu_{i}+$ $\mu_{j}+\mu_{k}$ of $\mu$. The same reasoning is applied if $a_{j k} \sim a_{k}$.

(b) MOPIC-2:

Let be $i, j, k \in N$ such that $\left\{\begin{array}{l}a_{i \vee j} T C_{P_{l}} a_{0} \\ a_{i j} P_{l^{\prime}} a_{k} \\ l>l^{\prime}\end{array}\right.$. By using equation 64, we have

$$
\mu_{i \vee j}>\mu_{i j}-\mu_{k}
$$

If $a_{i k} \sim a_{h}$ with $h \in[\{i, j\} \backslash i \vee j]$ then

$$
\mu_{i k}=\mu_{h} .
$$

Therefore we have

$$
\mu_{i \vee j}+\mu_{h}+\mu_{k}>\mu_{i j}+\mu_{i k} .
$$

A contradiction with the monotonicity constraint $\mu_{i j}+\mu_{i k} \geq \mu_{i \vee j}+$ $\mu_{h}+\mu_{k}$ of $\mu$. We use the same reasoning if we suppose $a_{j k} \sim a_{h}$. 


\section{(c) MOPIC-3:}

Let be $i, j, k \in N$ such that $\left\{\begin{array}{r}a_{k} T C_{P_{l}} a_{0} \\ a_{i j} P_{l^{\prime}} a_{i \vee j} \\ l>l^{\prime}\end{array}\right.$. By using equation 64, we have

$$
\mu_{k}>\mu_{i j}-\mu_{i \vee j}
$$

If we suppose $a_{i k} \sim a_{h}, h \in[\{i, j\} \backslash i \vee j]$, then

$$
\mu_{i k}=\mu_{h},
$$

and

$$
\mu_{i \vee j}+\mu_{h}+\mu_{k}>\mu_{i j}+\mu_{i k},
$$

by combining (71) and (72). This leads to a contradiction with the monotonicity constraint $\mu_{i j}+\mu_{i k} \geq \mu_{i \vee j}+\mu_{h}+\mu_{k}$ of $\mu$. We applied the same reasoning if we suppose $a_{j k} \sim a_{h}$.

The large number of necessary MOPI and MOPIC conditions suggest that, the final number of necessary and sufficient conditions, as simple as these conditions, for the representation of a cardinal information, could be very large.

\section{References}

[1] C. A. Bana e Costa, E. C. Correa, J.-M. De Corte, and J.-C. Vansnick. Facilitating bid evaluation in public call for tenders: A socio-technical approach. Omega, 30:227-242, 2002.

[2] C. A. Bana e Costa, J.-M. De Corte, and J.-C. Vansnick. On the mathematical foundations of MACBETH. In J. Figueira, S. Greco, and M. Ehrgott, editors, Multiple Criteria Decision Analysis: State of the Art Surveys, pages 409-437. Springer, 2005.

[3] C. A. Bana e Costa, F. Nunes da Silva, and J.-C. Vansnick. Conflict dissolution in the public sector: A case-study. European Journal of Operational Research, 130:388-401, 2001.

[4] L. Berrah and V. Clivillé. Towards an aggregation performance measurement system model in a supply chain context. Computers in Industry, 58(7):709-719, 2007.

[5] A. Chateauneuf. Ellsberg paradox intuition and Choquet expected utility. In G. Coletti, D. Dubois, and R. Scozzafava, editors, Mathematical models for handling partial knowledge in artificial intelligence, pages 1-20. Plenum Press, 1995.

[6] A. Chateauneuf, M. Grabisch, and A. Rico. Modeling attitudes towards uncertainty through the use of Sugeno integral. J. of Mathematical Economics, 44:1084-1099, 2008. 
[7] A. Chateauneuf and J.Y. Jaffray. Some characterizations of lower probabilities and other monotone capacities through the use of Möbius inversion. Mathematical Social Sciences, 17:263-283, 1989.

[8] A. Chateauneuf, R. Kast, and A. Lapied. Conditioning capacities and choquet integrals: The role of comonotony. Theory and Decision, 51(24):367-386, 2004.

[9] J.W. Chinneck. An effective polynomial-time heuristic for the minimum cardinality IIS set-covering problem. Annals of Mathematics and Artificial Intelligence, 17, 1996.

[10] G. Choquet. Theory of capacities. Annales de l'Institut Fourier, 5:131-295, 1953.

[11] V. Clivillé, L. Berrah, and G. Mauris. Quantitative expression and aggregation of performance measurements based on the MACBETH multicriteria method. International Journal of Production economics, 105:171$189,2007$.

[12] J.P. Doignon. Thresholds representation of multiple semiorders. SIAM Journal on Algebraic and Discrete Methods, 8:77-84, 1987.

[13] D. Ellsberg. Risk, ambiguity, and the Savage axioms. Quart. J. Econom., 75:643-669, 1961.

[14] T. Gajdos. Measuring inequalities without linearity in envy: Choquet integrals for symmetric capacities. J. of Economic Theory, 106:190-200, 2002.

[15] M. Grabisch. $k$-order additive discrete fuzzy measures and their representation. Fuzzy Sets and Systems, 92:167-189, 1997.

[16] M. Grabisch, J. Duchêne, F. Lino, and P. Perny. Subjective evaluation of discomfort in sitting position. Fuzzy Optimization and Decision Making, $1(3): 287-312,2002$.

[17] M. Grabisch and C. Labreuche. Fuzzy measures and integrals in MCDA. In J. Figueira, S. Greco, and M. Ehrgott, editors, Multiple Criteria Decision Analysis: State of the Art Surveys, pages 565-608. Springer, 2005.

[18] M. Grabisch and Ch. Labreuche. A decade of application of the Choquet and Sugeno integrals in multi-criteria decision aid. 4OR, 6:1-44, 2008.

[19] M. Grabisch, Ch. Labreuche, and J.-C. Vansnick. On the extension of pseudo-Boolean functions for the aggregation of interacting bipolar criteria. Eur. J. of Operational Research, 148:28-47, 2003.

[20] C. K. Hsee. The evaluability hypothesis: An explanation for preference reversals between joint and separate evaluations of alternatives. Organizational Behavior and Human Decision Processes, 67:242-257, 1996. 
[21] Ch. Labreuche and M. Grabisch. The Choquet integral for the aggregation of interval scales in multicriteria decision making. fuzzy sets and systems, $137: 11-26,2003$.

[22] O. L. Mangazarian. Nonlinear programming. New York: McGraw-Hill Book Company, 1969.

[23] T. Marchant. Towards a theory of MCDM: Stepping away from social choice theory. Mathematical Social Sciences, 45(3):343-363, 2003.

[24] B. Mayag, M. Grabisch, and C. Labreuche. A representation of preferences by the Choquet integral with respect to a 2 -additive capacity. accepted in Theory and Decision.

[25] P. Miranda, M. Grabisch, and P. Gil. p-symmetric fuzzy measures. Int. J. of Uncertainty, Fuzziness, and Knowledge-Based Systems, 10 (Suppl.):105$123,2002$.

[26] P. Miranda, M. Grabisch, and P. Gil. Axiomatic structure of $k$-additive capacities. Mathematical Social Sciences, 49:153-178, 2005.

[27] C. Mousset. Familles de structures de préférence non complètes. $\mathrm{PhD}$ thesis, University of Mons-Hainaut, Belgium, 2004.

[28] C. Mousset. Families of relations modelling preferences under incomplete information. European journal of operational research, 192(2):538-548, 2009 .

[29] C. Mousset and J.-C. Vansnick. About the representation of a precardinal information. Documents d'Economie et de Gestion de l'Université de MonsHainaut, 2005.

[30] T. Murofushi and S. Soneda. Techniques for reading fuzzy measures (III): interaction index. In 9th Fuzzy System Symposium, pages 693-696, Sapporo, Japan, May 1993. In Japanese.

[31] J.P. Pignon and Ch. Labreuche. A methodological approach for operational and technical experimentation based evaluation of systems of systems architectures. In Int. Conference on Software 83 Systems Engineering and their Applications (ICSSEA), Paris, France, December 4-6 2007.

[32] M. Pirlot and P. Vincke. Semiorders. Kluwer academic publishers, 1997.

[33] R.T. Rockafellar. Convex analysis. Princeton University Press, 1972.

[34] D. Schmeidler. Subjective probability and expected utility without additivity. Econometrica, 57(3):571-587, 1989.

[35] Alexander Schrijver. Theory of Linear and Integer programming. John Wiley and Sons, 1998. 
[36] Alexander Schrijver. Combinatorial Optimization, volume A. Springer, 2003.

[37] L. S. Shapley. A value for $n$-person games. In H. W. Kuhn and A. W. Tucker, editors, Contributions to the Theory of Games, Vol. II, number 28 in Annals of Mathematics Studies, pages 307-317. Princeton University Press, 1953.

[38] H. Simon. Rational choice and the structure of the environment. Psychological Review, 63(2):129-138, 1956.

[39] P. Slovic, M. Finucane, E. Peters, and D. G. MacGregor. The affect heuristic. In T. Gilovitch, D. Griffin, and D. Kahneman, editors, Heuristics and biases: the psychology of intuitive judgment, pages 397-420. Cambridge University Press, 2002.

[40] A. Tversky and D. Kahneman. Advances in prospect theory: cumulative representation of uncertainty. J. of Risk and Uncertainty, 5:297-323, 1992.

[41] J. Von Neuman and O. Morgenstern. Theory of Games and Economic Behavior. Princeton University Press, 1944.

[42] S. Weber. $\perp$-decomposable measures and integrals for Archimedean tconorms $\perp$. J. Math. Anal. Appl., 101:114-138, 1984.

[43] J. A. Weymark. Generalized Gini inequality indices. Mathematical Social Sciences, 1:409-430, 1981. 